\title{
Indicações da videotoracoscopia
}

\author{
Mirto N. Prandini*, Antônio P. F. Bonatelli*, Oswaldo I. Tella Jr. , Marco A. Herculano** \\ Disciplina de Neurocirurgia da UNIFESP, SP, e Disciplina de Neurocirurgia da Faculdade de Medicina de Jundiaí, SP
}

\section{RESUMO}

A videotoracoscopia (VT) vem apresentando grande destaque nos últimos anos, dentro do capítulo da "cirurgia minimamente invasiva". Durante quase um século, as indicações desse procedimento foram bastante limitadas. Com a evolução dos equipamentos eletrônicos e a produção de minivideocâmaras que permitem uma excelente visão das estruturas anatômicas, possibilitando a manufatura de endoscópios de tamanho bastante reduzido, foi possível a aplicação do método em praticamente todos os procedimentos em que a clássica toracotomia se fazia necessária. Os trabalhos recentes têm demonstrado uma série de vantagens da VT se comparadas com a toracotomia aberta. São citadas, como vantagens, a menor permanência do paciente em unidades de terapia intensiva, o período de internação hospitalar mais curto, a menor necessidade de analgésicos no pós-operatório, a redução na perda sangüínea, menores complicações pósoperatórias (hemotórax, empiema intratorácico, entre outras).

Apresentamos as indicações da VT para a cirurgia torácica, neurológica e ortopédica, assim como algumas das mais freqüentes complicações.

\section{PALAVRAS-CHAVE}

Videotoracoscopia. Cirurgia torácica videoassistida. Acesso anterior à coluna torácica.

\section{ABSTRACT}

Indications of video-thoracoscopy

In the area of "minimally invasive surgery", the video-thoracoscopy (V-T) is the one that has achieved the best results over the last years. After almost one century, when the indications for the procedure were very limited, the evolution of the electronic devices providing excellent vision of the anatomical structures and the miniaturization of the video camera, had expanded the role of $V-T$ to include a number of procedures that previously could be performed only by open approaches. Recent reports have demonstrated many advantages of $V-T$ if compared with classical open approaches: $V-T$ shortens the length of patients stay in the intensive care unit, as well as overall hospitalization time; analgesic requirement is reduced as is the blood loss; post-operative morbidity as empyema and atelectasis are reduced.

Indications for $V$ - $T$ in thoracic surgery, neurosurgery and orthopedics are presented as well as its complications and contraindications.

\section{KEYWORDS}

Video-thoracoscopy. Video-assisted thoracic surgery. Anterior approach to thoracic spine.

\section{Introdução}

Os procedimentos do capítulo da chamada "cirurgia minimamente invasiva" têm recebido uma atenção especial nos últimos anos, principalmente em decorrência dos avanços obtidos com os equipamentos eletrônicos que permitiram a redução substancial do tamanho das videocâmaras, sem que tenha havido prejuízo na qualidade de imagem obtida. Atualmente a tendência nas diversas especialidades é a redução das incisões e do afastamento das estruturas anatômicas nos procedimentos cirúrgicos, uma vez que, com isso, a recu- peração pós-operatória tem se mostrado bastante facilitada e com resultados cosméticos melhores. $\mathrm{Na}$ área da neurocirurgia, os resultados da cirurgia minimamente invasiva têm sido bastante animadores, assim como em diversas outras especialidades. A videotoracoscopia (VT) ocupa importante lugar neste novo campo.

\section{Histórico}

Aparentemente, o primeiro relato de um procedimento endoscópico remonta ao ano de 1806, quando Bozzin, utilizando-se de uma vela como fonte luminosa,

*Professor Adjunto da Disciplina de Neurocirurgia da UNIFESP - São Paulo, SP.

**Professor Assistente da Disciplina de Neurocirurgia da Faculdade de Medicina de Jundiaí, SP. 
executou um exame no interior da bexiga urinária, inventando, assim, o endoscópio ${ }^{13,14}$.

As difíceis condições técnicas praticamente limitavam o emprego do método e somente quase oitenta anos depois, em 1883, Newman utilizou uma lâmpada elétrica como fonte luminosa. Essa mudança possibilitou uma iluminação melhor, facilitando a visão do cirurgião, tornando o procedimento mais prático.

Os cistoscópios começaram a ter, desde então, uso mais difundido, até que, em 1910, Jacobaeus utilizou um desses aparelhos para biópsia na cavidade torácica como um elemento auxiliar no diagnóstico de tuberculose ${ }^{5}$. Foi o primeiro procedimento toracoscópico. A partir daí, sua aplicação foi ampliada, sofrendo, porém, muitas limitações, uma vez que, em casos de tumores do parênquima pulmonar, a possibilidade de sangramento tornava o seu uso bastante restrito. Por essas razões, durante muitos anos, predominou a clássica pleuroscopia e, eventualmente, biópsias de tumores intratorácicos, executados com algumas restrições e muitos cuidados, devido ao risco de o procedimento se transformar em toracotomias abertas ${ }^{8,12}$.

\section{Estado atual}

O desenvolvimento de "microchips" permitiu a confecção de microcâmaras que puderam ser embutidas em um endoscópio adaptado a uma fonte luminosa por fibra óptica e conectado a um equipamento de vídeo. Esse videoendoscópio permitiu a visão, com extrema nitidez, das estruturas da cavidade torácica, o que possibilitou a execução de praticamente todos os procedimentos, em que até então somente amplas toracotomias poderiam ser indicadas. Alguns autores consideram a videotoracoscopia como a mais significativa conquista na área da cirurgia torácica nos últimos vinte anos ${ }^{6-8}$.

\section{Indicações}

A chamada cirurgia "minimamente invasiva" tem revolucionado praticamente todas as áreas cirúrgicas. $\mathrm{O}$ uso dos endoscópios permite que os procedimentos sejam efetuados através de pequenas incisões, facilitando a recuperação dos pacientes, sendo, portanto, indicados em condições que exigem um maior cuidado pós-operatório.

Os endoscópios têm sido muito utilizados para execução das artroscopias na ortopedia, das laparoscopias pélvicas e abdominais, das cistoscopias urológicas, das ventriculoscopias cerebrais ${ }^{1}$ na neurocirur- gia, assim como importante auxiliar nas especialidades de otorrinolaringologia, otoscopia, etc $2,3,4,6,7,8$.

Para fins didáticos, as indicações do método são separadas em duas grandes vertentes ${ }^{1-4,6-14}$ :

1. para a cirurgia torácica;

2. para a neurocirurgia e ortopedia.

Ainda seria necessária uma separação dos procedimentos em diagnósticos e terapêuticos. Esquematicamente, teremos:

\section{Cirurgia torácica}

\begin{tabular}{l} 
Indicações mais aceitas da videotoracoscopia \\
\hline Diagnósticas \\
\hline Biópsia pulmonar \\
Biópsia pleural \\
Biópsia de processos expansivos do mediastino \\
Estagiamento de linfonodos da região aortopulmonar \\
Excisão de nódulos pulmonares indeterminados \\
Terapêuticas \\
\hline Esclerose de cavidade pleural infiltrado por metástases \\
Remoção de lesões pleurais benignas \\
Exérese de tumores pulmonares benignos \\
Simpatectomia para hiperidrose \\
Exérese de tumores do mediastino \\
Biópsia ou fenestração do pericárdio \\
Lobectomia pulmonar \\
Miomectomia do esôfago \\
Ligadura do ducto torácico em casos de quilotórax
\end{tabular}

\section{Neurocirurgia e ortopedia}

\begin{tabular}{l} 
Indicações mais aceitas da videotoracoscopia \\
\hline Diagnósticas \\
\hline Biópsia de corpo vertebral \\
Biópsia de processos expansivos paravertebrais \\
Terapêuticas \\
\hline Simpatectomia para hiperidrose e dores dos membros superiores \\
Descompressão anterior de corpo vertebral (trauma, tumor, infecção) \\
Remoção de disco intervertebral \\
Vertebrectomia e reconstrução vertebral \\
Artrodese vertebral \\
Correção de escoliose \\
Remoção de hemivértebra \\
Remoção de costela cervical e escalenotomia \\
Remoção de tumores paravertebrais
\end{tabular}

\section{Discussão}

O conceito de cirurgia "minimamente invasiva" tem sido ampliado para diversas áreas.

Kaiser $^{6}$ considera a VT como a mais importante conquista na área da cirurgia torácica nos últimos vinte anos. Para as cirurgias da região torácica anterior da 
coluna vertebral, a abordagem por meio da VT vem se constituindo como um elemento extremamente favorável, tendo, a cada dia, a área de atuação mais ampliada. Sem dúvida, por se tratar de uma nova abordagem, a curva de aprendizado tem sido mencionada como elemento extremamente importante ${ }^{2,4,6,7,12,15}$. É necessário o estágio em laboratório para que o cirurgião se torne familiarizado com o manuseio dos equipamentos, obtenha domínio do conhecimento das estruturas anatômicas, com nuances técnicas próprias para a execução dos procedimentos efetuados através de três ou quatro pequenos orifícios produzidos na caixa torácica e visualizados em uma tela bidimensional. Um importante elemento técnico é a intubação com sonda de "lúmen duplo", que permite que um dos pulmões seja colabado durante a cirurgia, ampliando o espaço que a caixa torácica, pela sua constituição, naturalmente já proporciona, conforme ressaltamos em publicação anterior ${ }^{13}$.

Os neurocirurgiões e ortopedistas modernos estão cada vez menos propensos a aceitar uma abordagem posterior para as afecções da coluna anterior. Durante décadas, tentou-se, com discutível sucesso, o desenvolvimento de abordagens do tipo póstero-lateral, com remoção de porções maiores ou menores do arco costal para acessar as regiões anteriores da coluna torácica. O campo cirúrgico, freqüentemente, mostrava-se insuficiente para a remoção total do processo patológico e a visão do campo operatório era bastante limitada. As amplas laminectomias efetuadas para as descompressões posteriores da medula espinhal, seja em casos de lesões traumáticas ou tumorais do corpo vertebral anteriormente situados, seja em protrusões dos discos intervertebrais, freqüentemente apresentavam grandes desapontamentos quanto aos resultados esperados. A abordagem anterior proporcionada pela técnica da VT permite que o cirurgião tenha campo visual quase total da coluna anterior e um campo de atuação extremamente favorável, se considerarmos os processos patológicos anteriormente situados ${ }^{1-3,6,9,10,12,15}$.

Se comparada com a toracotomia clássica, a VT permite um período menor de internação hospitalar, menor permanência em UTI, redução das dores incisionais, diminuição do volume de sangue administrado durante o ato cirúrgico, além de um melhor resultado cosmético da cicatriz cirúrgica ${ }^{1,2,6,12-14}$.

Segundo Landroneau e cols. ${ }^{9}, 10$, a comparação entre a morbidade pós-operatória da toracotomia e da VT mostrou que a VT propicia: tempo mais curto de permanência do paciente no ventilador, incidência menor de complicações como empiema e atelectasia, necessidade menor de reposição sangüínea e diminuição no tempo de permanência hospitalar.
Esses autores ainda relatam que os pacientes que foram submetidos a VT apresentaram menor desconforto pós-operatório e requereram menor número de bloqueios analgésicos, seja por infiltração de nervo intercostal, seja por analgesia peridural. Os autores ainda mencionam que o número de pacientes foi insuficiente para serem comprovados estatisticamente os aspectos observados quanto à melhor função respiratória nos casos de VT.

Rosenthal e cols. ${ }^{15}$ referem que a VT permite a remoção completa do disco intervertebral, com substancial redução no trauma cirúrgico, o que proporciona menores complicações pulmonares e as relacionadas à cicatriz cirúrgica. Permitindo um confinamento no leito menor, reduz os riscos de trombose venosa e do tempo de internação hospitalar, opinião esta compartilhada por outros autores ${ }^{3,8-10,12,15}$

Como qualquer procedimento cirúrgico, o método não está livre de complicações. De um modo geral, correspondem às complicações mais comuns presentes nas toracotomias clássicas. Podem ser citadas as atelectasias pulmonares, pneumotórax, hemotórax, pneumonia, arritmias cardíacas, quilotórax, lesões de estruturas viscerais, nervosas e vasculares do mediastino, entre outras menos comuns ${ }^{1-4,10-15}$.

De um modo geral, a presença de aderências pleurais, a dificuldade de o paciente suportar a intubação seletiva, a dificuldade no controle de sangramentos cirúrgicos, contra-indicam o método.

\section{Conclusões}

Entre os avanços técnicos nas especialidades de cirurgia torácica, neurocirurgia e ortopedia, a VT tem ocupado um lugar de destaque nos últimos anos. As vantagens proporcionadas pelo método têm incentivado, mais e mais, os cirurgiões a adotá-lo como prática rotineira. Uma vez familiarizados com esta abordagem, muitos consideram como ultrapassado o acesso posterior às lesões situadas anteriormente à medula espinhal. O fato de a caixa torácica apresentar uma consistência bastante firme, aliado à possibilidade de se poder colabar o pulmão homolateral, permite que se possa desfrutar de um espaço bastante amplo nos procedimentos efetuados nas regiões anteriores e laterais da coluna torácica. O crescente desenvolvimento tecnológico tem possibilitado a execução cada vez mais segura dos procedimentos em que o videoendoscópio é utilizado, melhorando, sob vários ângulos, os resultados pós-operatórios.

Por se tratar de método relativamente novo aos neurocirurgiões e ortopedistas, é recomendada a prática laboratorial antes da tentativa de execução de qualquer 
procedimento. Também, é considerada, como indispensável, a presença de cirurgião torácico como parte integrante da equipe, com a finalidade de se manusear melhor o pulmão e os vasos intratorácicos, ou mesmo para a eventualidade de o procedimento necessitar a transformação em toracotomia aberta.

\section{Referências}

1. COLTHARPWH, ARNOLD JH, ALFORD Jr WC: Videothoracoscopy: improved technique and expanded indications. Ann Thorac Surg 53:776-9, 1992.

2. COOPER JD: Perspectives on thoracoscopy in general thoracic surgery. Ann Thorac Surg 697-705, 1993.

3. DICKMAN CA, MICAN C: Thoracoscopic approaches for the treatment of anterior thoracic spinal pathology. BNI Q 2:4-11, 1996.

4. DICKMAN CA, ROSENTHAL D, KARAHALIOS DC: Thoracic vertebrectomy and reconstruction using a microsurgical thoracoscopic approach. Neurosurgery 38:279-84, 1996

5. JACOBAEUS HC: The practical importance of thoracoscopy in surgery of the chest. Surg Gynecol Obstet 32:289-96, 1922.

6. KAISER LR: Video-assisted thoracic surgery: current state of the art. Ann Surg 220:720-35, 1994.

7. KIRBY TJ, MACK MJ, LANDRENEAU RJ, RICE TW: Initial experience with video-assisted thoracoscopic lobectomy. Ann Thorac Surg 56:1248-57, 1993.
8. HOROWITZ MB, MOOSY JJ, JULIAN T: Thoracic discectomy using video-assisted thoracoscopy. Spine 19:1082-7, 1994

9. LANDRENEAU RJ, MACK MJ, HASEIRIGG SR: Videoassisted thoracic surgery: basic technical concepts and intercostal approach strategies. Ann Thorac Surg 54:800-9, 1992.

10. LANDRENEAU RJ, HAZELRIGG SR, MACK MJ: Postoperative pain-related morbidity video-assisted thoracic surgery versus thoracotomy. Ann Thorac Surg 56:1285-91, 1993.

11. MACK MJ, ARNOFF RJ, ACUFF TR: Present role of thoracoscopy on the diagnosis and treatment of diseases of the chest. Ann Thorac Surg 54:403-11, 1992.

12. OAKES DD, SHERCK JP, BRODSKY JB, MARK JBD: Therapeutic thoracoscopy. J Thorac Cardiovasc Surg 87:269-73, 1984

13. PRANDINI MN, TELLA OI: Thoracoscopic approaches in the spine. Contemp Neurosurg 23:1-5, 2001.

14. PRANDINI MN, TELLA OI: Thoracoscopic approaches in the spine.Video-assisted thoracic surgery. Contemp Spine Surg 6:43-7, 2002

15. ROSENTHAL D, ROSENTHAL R, SIMONE A: Removal of a protruded thoracic disc using microsurgical endoscopy. Spine 1087-93, 1994.

Original recebido em outubro de 2002

Aceito para publicação em dezembro de 2002

\section{Endereço para correspondência:}

Mirto N. Prandini

Rua dos Crisântemos, 117

CEP 04049-020 - São Paulo, SP 